Pis'ma v ZhETF

\title{
On the problem of catastrophic relaxation in superfluid ${ }^{3} \mathrm{He}-\mathrm{B}$
}

\author{
Yu. M. Bunkov ${ }^{a}$ 1), V.S.L'vov ${ }^{b, c}$, G. E. Volovik ${ }^{c, d}$ \\ ${ }^{a}$ Centre de Recherches sur les Très Basses Températures, CNRS, BP166, 38042, Grenoble, France \\ ${ }^{b}$ Department of Chemical Physics, The Weizmann Institute of Science, Rehovot 76100, Israel \\ ${ }^{c}$ Low Temperature Laboratory, Helsinki University of Technology, P.O.Box 2200, FIN-02015, HUT, Finland \\ ${ }^{d}$ Landau Institute for Theoretical Physics RAS, Kosygina 2, 117334 Moscow, Russia \\ Submitted 15 August 2006 \\ Resubmitted
}

\begin{abstract}
In this Letter we discussed the parametric instability of texture of homogeneous (in time) spin precession, explaining how spatial inhomogeneity of the texture may change the threshold of the instability in comparison with idealized spatial homogeneous case, considered in our JETP Letter 83, 530 (2006). This discussion is inspired by critical comment of I.A. Fomin (cond-mat/0606760) related to the above questions. In addition we considered here results of direct numerical simulations of the full Leggett-Takagi equation of motion for magnetization in ${ }^{3} \mathrm{He}-\mathrm{B}$ and experimental data for magnetic field dependence of the catastrophic relaxation, that provide solid support of the theory of this phenomenon, presented in our 2006 JETP Letter.
\end{abstract}

PACS: $67.57 . \mathrm{Lm}, 76.50 .+\mathrm{g}$

Introduction. This Letter is inspired by critical comment [1] on our JETP Letter "Solution of the problem of 'catastrophic relaxation of homogeneous spin precession in superfluid ${ }^{3} \mathrm{He}-\mathrm{B}$ " 2]. The self sustained and longlived spin precession with the coherent phase across the 'whole precessing domain - the Homogeneously Precessing Domain (HPD) - is a unique feature of superfluid ${ }^{3} \mathrm{He}-\mathrm{B}$. This phenomenon bears all the ingredients of spin superfluidity [3. Later it was found that at low 'temperatures, below $\sim(0.4-0.5) T_{c}$, the spin superfluidity experiences abrupt instability, called the catastrophic relaxation [4.

After many attempts it was finally recognized that the origin of the catastrophe is the parametric (Suhl) instability 5, 2. Two competing contributions to the increment of the parametric instability were suggested:

$$
V(\boldsymbol{r})=V_{\mathrm{SF}}(\boldsymbol{r})+V_{\mathrm{BLV}}(\boldsymbol{r}) .
$$

The "intrinsic" contribution $V_{\mathrm{SF}}$, suggested in the Sourovtsev-Fomin (SF) Ref. [5], is due to the anisotropy of spin-wave velocity and it comes from the region where the configuration of spin and orbital vectors $\boldsymbol{S}$ and $\boldsymbol{L}$ is canonical, the so-called the Brinkman-Smith (BS) mode of precession. Suggested in our Bunkov-L'vov-Volovik (BLV) Ref. 2] contribution $V_{\mathrm{BLV}}$ is due the oscillating spin-orbit energy and it only comes from the region where precession deviates from the BS mode.

\footnotetext{
1) e-mails: victor.lvov@weizmann.ac.il,
}

yuriy.bunkov@grenoble.cnrs.fr, volovik@boojum.hut.fi
Comment 1] clarifies aspects of the problem that may cause misunderstanding and thus require more detailed explanation and even further development. This is the subject of our Letter, which is organized as follows. Sections 1-3 are devoted to questions, arose in [1, which are mainly related to the problem of parametric instability in the case of the spatially inhomogeneous precession. In Sec. 4 we consider extension of our theory to other precessing states. In Secs. 5 and 6 we discuss results of direct numerical simulations of the full Leggett-Takagi equations for magnetization and of laboratory study of the magnetic field dependence of the catastrophic relaxation, that provide additional support of our theory of this phenomena, presented in Ref. [2, and demonstrates that the SF mechanism of the instability [5] is in contradiction with the experiments.

1. Stability of the basic reference state. This is the first question of Com. 1] that is useful to clarify. As stated in [1, the spin-orbit potential energy as a function of $S_{z}$ has concave or the convex form shape, depending on orientations of $\boldsymbol{L}$ and $\boldsymbol{S}$ and therefore in some cases the spatially homogeneous precession can be unstable [6]. For example, the conventional homogeneous precession is unstable in ${ }^{3} \mathrm{He}-\mathrm{A}$ [7]. However, this is not applicable to a "texture" with some profile of the orientations of the orbital and spin moments, $\boldsymbol{L}$ and $\boldsymbol{S}$, that realizes the minimum of the sum of the gradient and the spin-orbit energies (see Ref. [8] for the texture in slab geometry and Ref. [9] for the texture under discussion). The texture is stable with respect 
to perturbations with the same rotation frequency $\omega_{L}$. This texture, shown in Fig. 1 of our Ref [2], serves as the basic reference state, stability of which with respect to parametric excitation of the spin waves with frequency $\omega_{s}( \pm \boldsymbol{k})=\omega_{L} / 2$, was studied in our Ref. [2]. Notice again, that the texture is stable with respect to perturbations with the frequency $\omega_{L}$. This is the answer to the first question of [1]: "Why the same mechanism which is responsible for the instability of the uniform precession in ${ }^{3} \mathrm{He}-\mathrm{A}$ can be disregarded?".

2. Parametric instability in spatially inhomogeneous media. The second statement of Com. [1] is trivial: because the spatial variations of the parametric instability increment $V$ "is not small . . , two ways of averaging $[\exp (\langle V\rangle t)$ and $\langle\exp (V t)\rangle]$ can give very different results". The relevant question here: "What is the adequate way of averaging of the instability increment (if it exists)?" To answer this question one has to describe parametric instability in spatially inhomogeneous media.

This problem has been discussed in various physical situations and is presented in many books, for example in Sec. 6.5.2 of monograph [10, where explicit expressions for the instability increment for different types of spatial inhomogeneities are derived. Without going into details we can say that the main physical message of this study is that for weakly decaying parametric waves (which is our case) the threshold of the parametric instability can be estimated with a good accuracy from the total energy balance in the sample (cell, in our case). Namely, at the threshold the total energy influx into the system

$$
W_{+} \propto \int V[\boldsymbol{r}, \boldsymbol{k}(\boldsymbol{r})] d \boldsymbol{r},
$$

has to be equal to the total rate of the energy dissipation $W_{-}$. With the same prefactor as in Eq. (2)

$$
W_{-} \propto \int \gamma[\boldsymbol{k}(\boldsymbol{r})] d \boldsymbol{r}
$$

where $\gamma(\boldsymbol{r})$ is the damping rate of the parametric waves.

The physical reason is that under wave propagation in weakly inhomogeneous media its frequency serves as the adiabatic invariant. This means that the wave frequency is independent of the position, while the wavevector $\boldsymbol{k}(\boldsymbol{r})$ changes accordingly to the dispersion law $\omega(\boldsymbol{k}, \boldsymbol{r})=$ const. Because of that, even propagating in inhomogeneous media, the waves with

$$
\omega[\boldsymbol{k}(\boldsymbol{r}), \boldsymbol{r}]=\omega_{L} / 2,
$$

stay in parametric resonance with the pumping (homogeneous in frequency, but spatially inhomogeneous spin precession). In other words, if the mean-free pass of the wave exceeds the cell size, the entire cell can be considered as the resonator with some parametrical mode, which is locally close to the planar spin wave. Under this conditions, the threshold can be well estimated from the condition:

$$
\int V[\boldsymbol{r}, \boldsymbol{k}(\boldsymbol{r})] d \boldsymbol{r}=\int \gamma[\boldsymbol{k}(\boldsymbol{r})] d \boldsymbol{r} .
$$

Accuracy of this estimate is about $\pm(10 \div 30) \%$, and related with ignoring in Eq. (5) the spatial variation of the actual profile of the parametric waves (for more details, see i.e. Ref. [10]).

In experimental conditions, (see Fig. 1 in our Ref. 2]) one roughly says, that the fraction of the cell volume (near the wall), with essential deviation from the planar geometry is about of a half of the total volume. This is the volume, where the energy pumping is dominating [i.e. the integral in the LHS of Eq. (5)]. In this region, in agreement with the the third comment of [1], the spin-wave vectors are relatively small and thus one can neglect the wave damping. The wave damping [the integral in the RHS of Eq. [5]] is dominated by the central part of the cell, where wave vectors are sufficiently large. Accordingly, the total energy balance in the cell is kept by the spatial energy flux from the near-wall region to the central part of the cell. Under this conditions, the threshold Eq. (5) can be roughly rewritten in the simple manner:

$$
\mathcal{V}_{V} \max V[\boldsymbol{r}, \boldsymbol{k}(\boldsymbol{r})] \simeq \mathcal{V}_{\gamma} \max \gamma[\boldsymbol{k}(\boldsymbol{r})],
$$

in spite of the fact, that the energy pumping and the energy damping are dominating in the different regions of the cell. Here $\mathcal{V}_{\gamma}$ and $\mathcal{V}_{V}$ are the corresponding effective volumes which depend on texture. As follows from the analytical and numerical simulations, these volumes do not depend on the value of magnetic field (see below) and in the considered cell are roughly equal. This means that

$$
\max V[\boldsymbol{r}, \boldsymbol{k}(\boldsymbol{r})] \simeq \max \gamma[\boldsymbol{k}(\boldsymbol{r})],
$$

estimate, used in our Ref. [2].

3. Wave damping in the near-wall region. In the third complain of [1] we have been instructed how to estimate from Eq. (4) wave vector of $\boldsymbol{k}(\boldsymbol{r})$. Presented estimate shows that near the wall wave vectors and, consequently, wave damping is small with respect of the central region with the conclusion that we miss a factor about 20 in our estimate of the parametric threshold. The misunderstanding of Ref. [1] in this point is related to the question: "How the value of the wave damping 
in the near-wall region effects on the threshold of the parametric excitation in the cell?" We hope that our explanation in previous Sec. 2 is clear enough to realize that the energy balance in the system of weakly decaying waves has to be discussed rather globally, for the entire cell, then locally, point-wise, as presented in Comment [1. Therefore, the estimates of the spin-wave vectors in the near-wall region, made in Com. 1], being reasonable itself, are irrelevant to the problem at hand. The same we can say about the statement, made in 1, concerning "additional factor $\approx 20$ in the RHS of Eq. (28)" in our Ref. 2]. As we explained in Sec. 2, the possible inaccuracy of our estimate of the threshold [2] does not exceed $(20 \div 30) \%$.

4. Generalization on other precessing states. To conclude the subject of the spatial inhomogeneity we mention, that the precession under discussion - the HPD - is a very specific precessing state due to its unique symmetry. Only in the case of the BrinkmanSmith mode the spin-orbit interaction does not contribute to the amplitude of the parametric instability $V$, and thus the parametric excitation by the BLV mechanism requires the existence of the texture.

However, there are many other modes of spin precession in ${ }^{3} \mathrm{He}$, for which the spin-orbit potential energy as a function of $S_{z}$ has also the concave form shape. These are: the so-called HPD2 in ${ }^{3} \mathrm{He}-\mathrm{B}$ [1]; the special mode of precession in ${ }^{3} \mathrm{He}-\mathrm{A}$ [12]; the precession at one half of equilibrium magnetization and at almost zero magnetization observed in ${ }^{3} \mathrm{He}-\mathrm{B}[13$; etc. As distinct from the HPD based on the Brinkman-Smith mode, in all these modes the spin-orbit energy is oscillating, and thus it produces the non-zero contribution to $V$ even in case of the spatially homogeneous precession. This means that for all these modes of spatially homogeneous precession, the BLV mechanism, based on the spin-orbit energy, will compete with the SF mechanism even at moderate magnetic fields.

Notice, that some of the predicted HPD modes still have not been observed or identified. Now with our knowledge of the parametric instability mechanisms and their dependence on different parameters, we are able to find the region of parameters (magnetic field, temperature, angles of precession, superfluid velocity, etc.) where all the conditions for the stability of precession are satisfied.

Now we are coming to the philosophical discussion, open in Com. 1] of "validity of an idealization for a particular experimental set-up" and of acceptance of "generalization of theory in a way, that makes possible its application to wider class of experimental condi- tions". Obviously, a success of "idealization" or "generalization", used in a theory, depends on an experience, physical intuition, taste and courage of its authors, that are different for different investigators. We hope that we made it clear in Secs. 1-3, that what it seems for the author of Com. [1 "ambiguous assumptions and non justified approximations" have indeed clear sense and well established physical background.

Nevertheless we are happy, using the discussion with Com. [1], to provide our theory of catastrophic relaxation [2] with additional support from the direct numerical simulations of full Leggett-Takagi equations 14 (in the next Sec. 5) and especially from the laboratory study of magnetic field dependence of the temperature of catastrophic relaxation in ${ }^{3} \mathrm{He}$, Sec. 6. Note that most of the complications related to the spatially inhomogeneous precession disappear at high magnetic field, where the wave vector $k$ is almost constant across the cell.

5. Discussion of numerical experiment. Onedimensional numerical simulations of the LeggettTakagi equations were made in Ref. [14. It was found that in the viscous limit, i.e. at sufficiently high temperature, the Brinkman-Smith configuration takes place in the central part of the cell being disturbed by the periodic perturbations in angle $\theta$ coming from the peripheral region, where the vector $\boldsymbol{L}$ considerably deviates from the Brinkman-Smith configuration. This observation confirms our statement, made in Sec. 1, that the spatial inhomogeneous texture is stable, providing minimum of the spin-orbit and gradient energies at given boundary conditions and can not be destroyed by any relaxation processes.

At further cooling of the cell, the BS configuration is more and more disturbed, and at some at some threshold temperature $T_{\mathrm{th}}$ the catastrophe occurs: the exponential growth of standing spin waves [with frequency $\left.\omega_{L} / 2\right]$ starts in the peripheral region and propagates to the central part, taking energy from the homogeneous precession and finally destroying it. This picture is in full agreement with the parametric instability of the inhomogeneous precession, studied in Refs. 2, [5].

Let us remind that there are two competing contributions to the increment of the parametric instability in Eq. (11). The "intrinsic" contribution $V_{\mathrm{SF}}$ comes from the whole sample; and the contribution $V_{\mathrm{BLV}}$ which is due the oscillating spin-orbit energy and it only comes from the regions where the configuration deviates from the Brinkman-Smith mode. In the one-dimensional geometry, used in the numerics 14, the SF contribution is suppressed in the central region because the increment is zero for the waves radiated in the direction perpendic- 


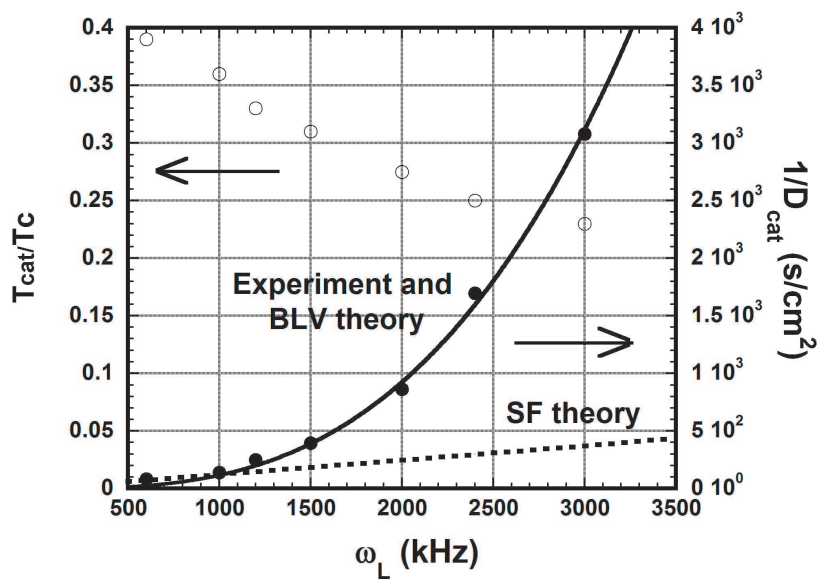

The experimental data for the temperature of catastrophic relaxation as a function of the NMR frequency at 31 bar ( o ), left scale, and the estimated value of the inverse spin diffusion coefficient, ( ) right scale. Solid line - BLV theoretical estimation [2], dashed line - SF results 5 .

ular to $\boldsymbol{L}$ (cf. Eq.(26) of Ref. [5] and Eq.(25) in Ref. [2]. This means that while these particular numerical simulations support the process discussed by the BLV theory [2]), they cannot either support or disregard the relevance of SF contribution at given parameters of the simulations.

6. Magnetic field dependence: SF vs. BLV competition. To find out which contribution is dominating in real experimental situations, we will compare both contributions to the increment (11) 2, 5] directly with laboratory experiments. The BLV arguments [2] demonstrate that in the moderate magnetic fields the $V_{\mathrm{BLV}}$ contribution is dominating. Both groups [1, 2] agree, that according to the calculations of Ref. [5], the SF contribution $V_{\mathrm{SF}}$ dominates at large magnetic field, when $\omega_{L} \gg \Omega_{L}$. To see that this is really the case, we examine here experimental data on magnetic field dependence, that include the region of higher magnetic fields.

The most relevant for this goal data are provided by the Cornell [15] and Grenoble [16] experiments, where the temperature of catastrophic relaxation has been measured as a function of magnetic field, see Fig. 1. Open circles in Fig. 1 demonstrate the temperature $T_{\text {cat }}\left(\omega_{L}\right)$ of the catastrophic relaxation as a function of the magnetic field (or Larmor frequency $\omega_{L}$ ) at 31 bar [15. The main effect of the temperature is to provide the dissipation which damps the parametric instability via the spin-diffusion mechanism 5. That is why the relevant physical quantity is the spin-diffusion coefficient $D\left(T, \omega_{L}\right)$, rather than the temperature itself, and we must convert the temperature to diffusion. According to Ref. [17, in the ballistic regime considered here when $\omega_{L} \tau(T) \gg 1$, the spin diffusion $D\left(T, \omega_{L}\right) \sim 1 / \omega_{L} \tau(T)$, where $\tau(T)$ is a quasiparticles scattering time, which grows exponentially with cooling. The experimental temperature dependence of the spin diffusion is obtained from measurements of diffusion through the HPD boundary [18.

Combining these results with $T_{\text {cat }}\left(\omega_{L}\right)$ from Fig. 1 one obtains the spin diffusion at which the catastrophic relaxation occurs as a function of $\omega_{L}$ : solid circles in Fig. 1 show $D_{\text {cat }}^{-1}\left(\omega_{L}\right)$. The experimental points fit excellently the cubic dependence

$$
\frac{1}{D_{\text {cat }}^{\mathrm{BLV}}}=\frac{\omega_{L}^{3}}{4 \bar{a} \Omega_{L}^{2} c_{\|}^{2}} \propto \omega_{L}^{3},
$$

obtained within the BLV mechanism \{see Eqs. (23) and (24) in Ref. 2]\}.

For the quantitative comparison of the experiment for $\omega_{L}$ dependence of $1 / D_{\text {cat }}$ with Eq. (8) we shall take the experimental values of the parameters $\Omega_{L}, c_{\|}$ and use the parameter $\bar{a}$, which characterizes the $\boldsymbol{L}$ texture, as a fitting parameter. In the considered region of temperature all these parameters are slow functions of temperature. We shall use the data at $1 \mathrm{MHz}$ and $T=0.35 T_{\mathrm{c}}$, where reliable experimental data exist. From Ref. [19] we can estimate $\Omega_{L}^{2}=10^{11} \mathrm{~Hz}^{2}$; while from measurements of different modes of HPD oscillations 20] with pressure scaling by Fermi velocity, we find $c_{\|}^{2}=1.5 \times 10^{6} \mathrm{~cm}^{2} / \mathrm{s}^{2}$. By introducing these values into Eq. (8), one obtains $\bar{a}=0.07$, which is in good agreement with the theoretical estimation of $\bar{a} \approx 0.1$ for 6 bar, made in Ref. 2].

In the current consideration it is important that the parameter $\bar{a}$ does not depend on magnetic field. The reason for that is that according to the Ref. 9, the characteristic length scale of the near wall region is $\sim c / \sqrt{\omega\left(\omega-\omega_{L}\right)}$. It is typically about $c / \Omega_{L}$, and thus does not depend on $\omega_{L}$.

Next step is to compare the experimental results with the SF contribution [5]. It reads:

$$
\frac{1}{D_{\text {cat }}^{\mathrm{SF}}}=\frac{\omega_{L}^{2}}{2 \lambda_{\max } c_{\|}^{2}} \propto \omega_{L} .
$$

Here we accounted for that $\lambda_{\max }$ near the HPD boundary is equal $0.016 \omega_{L}$ and therefore the field dependence of $1 / D_{\text {cat }}^{\mathrm{SF}}$ should be linear. As one see in Fig. 1 this clearly contradicts to the experiment. Furthermore, if we plot the value of $1 / D_{\text {cat }}^{\mathrm{SF}}$, we find (Fig. 1) that the 
SF result agrees with the experiment only in the region of NMR of $500-1000 \mathrm{MHz}$. At higher fields the theoretical value of diffusion, at which catastrophic relaxation with the SF mechanism should occur, definitely disagrees with the experiments.

Discussion. According to our theoretical analysis, in moderate magnetic fields, i.e. at $\omega_{L}$ smaller than about $1 \mathrm{MHz}$, the BLV contribution the parametric instability of HPD is dominating. At these fields the effect of spatially inhomogeneity on the BLV mechanism is essential, and we clarified in Secs. 2 and 3 the corresponding points, rouse in Com. 1. Most of the complications related to the issue of spatial inhomogeneity do not arise at high magnetic fields, where $\omega_{L} \gg \Omega_{L}$, and the wave vector $k$ is (almost) homogeneous along the cell. However, we expected that at such a high field the BLV contribution is subleading, while the SF contribution dominates, if $\omega_{L}^{2}>10 \Omega_{L}^{2}$. The same opinion was expressed in Com. [1, where it was stressed that the stronger magnetic fields have to be used for the experimental investigation of the "intrinsic" SF mechanism of catastrophic relaxation.

On the contrary, the surprising experimental fact is that the magnetic field dependence of the catastrophic relaxation demonstrates that even up to a rather high field, when $\omega_{L}^{2} \sim 100 \Omega_{L}^{2}$, it is still quantitatively and qualitatively described by the BLV contribution to the parametric instability. Moreover, the magnetic field dependence is in striking disagreement with the SF contribution. The matter of the fact is that the SF contribution does not show up in these experiments at all.

We do not think that explanation of this fact is related to possible calculational mistakes in the analytics, presented in Ref. [5]. We feel that reason(s) for the obvious qualitative disagreement between the $\mathrm{SF}$ analysis and experiment is deeper and may be related to possible violations of the SF approach in the region of large $k$ vectors.

To make long story short, the BLV mechanism of the parametric instability gives quantitative description of the present experiments for any values of $\omega_{L}$ used, while the SF contribution, that seems to be the leading one (for large $\omega_{L}$ ), is absent in the experiments by unknown reason. This is the main puzzle; its solution requires the further theoretical, numerical and experimental efforts, that are beyond the scope of this Letter.

Acknowledgements. We acknowledge the critical comments by I.A. Fomin [1, that inspire us for further clarification and development of our theory of catastrophic relaxation in superfluid ${ }^{3} \mathrm{He}-\mathrm{B}$, presented in this Letter. This work was done as the result of collaboration in the framework of the ESF Program COSLAB, the Large Scale Installation Program ULTI of the European Union (contract number: RITA-CT-2003-505313) and the project ULTIMA of the "Agense National de Recherche", France. The work was also supported in part by the Russian Foundation for Fundamental Research and the US-Israel Binational Science Foundation.

1. I.A. Fomin, Comment on "solution of the problem of catastrophic relaxation of homogeneous spin precession in superfluid ${ }^{3}$ He-B", cond-mat/0606760

2. Yu. M. Bunkov, V.S. L'vov and G.V. Volovik, Solution of the problem of catastrophic relaxation of homogeneous spin precession in superfluid ${ }^{3} \mathrm{He}-\mathrm{B}$ ", Pis'ma ZhETF, 83, 624-629 (2006) [JETP Lett. 83, 530-535 (2006)], cond-mat/0605386

3. See review: Yu.M. Bunkov, "Spin Supercurrent and Novel Properties of NMR in ${ }^{3} \mathrm{He}$ ", in "Progress in Low Temp. Physics" 14, 69, ed. W. Halperin, Elsevier (1995).

4. Yu.M. Bunkov, V.V. Dmitriev, Yu.M. Mukharsky, J. Nyeki and D. A. Sergatskov, Europhys. Lett., 8, 645 (1989).

5. E.V. Sourovtsev, I.A. Fomin, Pis'ma ZhETF, 83, 479 (2006).

6. I.A. Fomin, ZhETF, 78 2392, (1980) [JETP 511203 (1980)].

7. I.A. Fomin, Pis'ma ZhETF 30, 179 (1979) [JETP Lett. 30, 164 (1979)].

8. L.R. Corruccini and D.D. Osheroff, Phys. Rev. B 17, 126 (1978).

9. Yu. M. Bunkov, O. D. Timofeevskaya and G. E. Volovik, Phys. Rev. Lett. 73, 1817 (1994).

10. V.S. L'vov, Wave Turbulence Under Parametric Excitation, (Springer-Verlag, 1994)

11. Yu. M. Bunkov and G.E. Volovik, ZhETF 103, 1619 (1993) [JETP 76, 794, (1993)].

12. Yu. M. Bunkov, and G.E. Volovik, Europhys. Lett. 21, 837-843 (1993).

13. V.V. Dmitriev, I.V. Kosarev, M. Krusius, D.V. Ponarin, V.M.H. Ruutu, and G.E. Volovik, Phys. Rev. Lett. 78, 86 (1997).

14. Yu. M. Bunkov, V.L.Golo, O. D. Timofeevskaya Czech. J. Phys., 46, 213 (1996), J. Low. Temp. Phys., 137, 625 (2004).

15. D.A. Geller, D.M. Lee, Phys. Rev. Lett. 85, 1032 (2000).

16. Yu. M. Bunkov, J. Low. Temp. Phys. 135, 337 (2004).

17. D. Einzel, J. Low. Temp. Phys. 84, 321 (1991).

18. Yu.M. Bunkov, V.V. Dmitriev, A. M. Markelov, Yu. M. Mukharsky and D. Einzel, Phys. Rev. Lett. 65, 867 (1990).

19. P.J. Hakonen, M. Krusius, M.M. Salomaa, et al., J. Low. Temp. Phys. 76, 225 (1989).

20. Yu.M. Bunkov, V.V. Dmitriev and Yu.M. Mukharsky Physica B 178, 196 (1992). 